\title{
La no-ficción latinoamericana: del documental interactivo al documental transmedia
}

\author{
FERNANDO IRIGARAY \\ Universidad Nacional de Rosario, Argentina \\ fgirigaray@gmail.com \\ https://orcid.org/0000-0003-1270-8148
}

\author{
Anahí Lovato \\ Universidad Nacional de Rosario, Argentina \\ lovatoanahi@gmail.com \\ https://orcid.org/0000-0003-1451-2851
}

\section{Latin American non-fiction: from interactive to transmedia documentary}

\section{RESUMEN ABSTRACT}

En las últimas décadas, diversos procesos de convergencia digital y tecnológica hicieron posible la mutación de los formatos narrativos y la aparición de nuevas especies en el campo de la comunicación. Las narrativas de no-ficción no resultaron ajenas a estas transformaciones. Muy por el contrario, muchos proyectos documentales y periodísticos comenzaron a asumir formas interactivas y transmediales, experimentando con múltiples lenguajes, desarrollando estrategias participativas e historias que se expanden en diversas plataformas y soportes. En Latinoamérica, el campo de la no-ficción exhibe profundas raíces, sostenidas en la pluma de sus grandes cronistas y en las producciones audiovisuales de sus escuelas de documental político y social. Esta huella de identidad puede rastrearse igualmente en los formatos interactivos, en ocasiones mencionados como webdocs, idocs, documentales multimedia interactivos e incluso en proyectos transmedia, de corte documental o periodístico. El documental interactivo y transmedia en América Latina tiene ya más de dos décadas de desarrollo y consolidación. A lo largo de su extensa geografía es posible reconocer algunos polos de producción muy activos en la búsqueda de formatos innovadores y en la experimentación con nuevas tecnologías para narrar historias del orden de lo real. El presente número de Hipertext.net propone una mirada crítica que permita mapear el estado de la cuestión,

historizando sus producciones, analizando sus características intrínsecas, revisando sus experiencias de diseño narrativo, circulación y consumo, e identificando asimismo los desafíos y oportunidades que se presentan para la no-ficción interactiva y transmedia en esta región del mundo.

PALABRAS CLAVE

Documental transmedia; No-ficción; Latinoamérica; Documental interactivo; Innovación mediática.
In recent decades, several processes of digital and technological convergence have made possible the mutation of narrative formats and the appearance of new forms in the field of communication. Non-fiction narratives were not alien to these transformations. On the contrary, many documentary and journalistic projects began to assume interactive and transmedia formats and involved experimentation with multiple languages, the development of participatory strategies and stories that spread across different platforms and media. In Latin America, non-fiction has deep roots, sustained in the writing of its great chroniclers and in the audiovisual productions of its schools of political and social documentary. This stamp of identity can also be traced in interactive formats, sometimes referred to as webdocs, idocs, interactive multimedia documentaries and even in documentary or journalistic transmedia projects. Interactive and transmedia documentary in Latin America has now been under development and in consolidation for over two decades. Throughout the continent's extensive geography, it is possible to recognize some production poles that are very active in the search for innovative formats and in experimentation with new technologies to tell stories of the order of reality. This issue of Hipertext.net proposes a critical perspective from which to map the state of the art while historicizing documentary productions, analyzing their intrinsic characteristics, reviewing their experiences of narrative design, circulation, and consumption, and identifying the challenges and opportunities that arise for interactive and transmedia non-fiction in this region of the world.

\section{KEYWORDS}

Transmedia documentary; Non-fiction; Latin American; Interactive documentary; Media innovation. 


\section{La no-ficció llatinoamericana: del documental interactiu al documental transmèdia}

\section{RESUM}

En les últimes dècades, diversos processos de convergència digital i tecnològica han fet possible la mutació dels formats narratius i l'aparició de nous espècimens en el camp de la comunicació. Les narratives de no-ficció no s'han mantingut alienes a aquestes transformacions. Molt al contrari, molts projectes documentals i periodístics han començar a assumir formes interactives $\mathrm{i}$ transmedials, experimentant amb múltiples llenguatges, desenvolupant estratègies participatives i relats que s'expandeixen en diverses plataformes i suports. A Llatinoamèrica, el camp de la no-ficció compta amb profundes arrels, sostingudes en la ploma dels seus grans cronistes i en les produccions audiovisuals de les seves escoles de documental polític i social. Aquesta empremta d'identitat es pot rastrejar en els formats interactius, anomenats també com webdocs, iDocs, documentals multimèdia interactius $i$ fins $i$ tot en projectes transmèdia, de tall documental o periodístic.

El documental interactiu i transmèdia a Amèrica Llatina té ja més de dues dècades de desenvolupament i consolidació. Al llarg de la seva extensa geografia és possible reconèixer alguns pols de producció molt actius en la recerca de formats innovadors i en l'experimentació amb noves tecnologies per narrar històries de l'ordre de la realitat. El present número de Hipertext.net proposa una mirada crítica que permeti cartografiar l'estat de la qüestió, revisar històricament les seves produccions, analitzant les seves característiques intrínseques, revisant les seves experiències de disseny narratiu, circulació i consum, així com identificant els desafiaments i oportunitats que es presenten per a la no-ficció interactiva i transmèdia en aquesta regió del món.

\section{PARAULES CLAU}

Documental transmèdia; No-ficció; Llatinoamèrica;

Documental interactiu; Innovació mediàtica.
En las últimas décadas, diversos procesos de convergencia digital y tecnológica hicieron posible la mutación de los formatos narrativos y la aparición de nuevas especies en el campo de la comunicación. Las narrativas de no-ficción no resultaron ajenas a estas transformaciones. Muy por el contrario, muchos proyectos documentales y periodísticos comenzaron a asumir formas interactivas, inmersivas y transmediales, experimentando con múltiples lenguajes, desarrollando estrategias participativas e historias que se expanden en diversas plataformas y soportes.

Aunque excede los objetivos y alcances de este texto, es conveniente puntualizar que el concepto América Latina puede diferir en cuanto límites indiscutidos. Para algunos se extiende desde el sur del Río Bravo al Cabo de Hornos teniendo en común la lengua española y portuguesa. Para otros trasciende estos territorios e incluye comunidades que hablan idiomas de raíz latina como el francés. Desde una mirada decolonial, se denuncia que estas posturas excluyen de forma manifiesta a los pueblos originarios, como si la historia de esta parte del mundo hubiera comenzado en 1492. Ciertamente, se trata de un continente en disputa, no tan solo en lo político y económico, sino fundamentalmente en lo simbólico.

En este territorio en disputa, el campo de la no-ficción exhibe profundas raíces, sostenidas en la pluma de sus grandes cronistas y en las producciones audiovisuales de sus escuelas de documental político y social. Una tradición ligada a los procesos emancipatorios y a las narrativas de denuncia recorre la espina dorsal latinoamericana. Esta tradición abarca tanto procesos macrosociales como historias mínimas, incluyendo un amplio abanico que va desde las meta-narrativas de la liberación a las narraciones subjetivas, del cine directo y testimonial al poético y al realismo mágico. Desde la década de los '60, sin embargo, más allá de las formas y las temáticas, América Latina mantuvo, en general, una línea que trata la desigualdad y las injusticias políticas y sociales y visibiliza a los excluidos.

Fernando Birri (1964), cineasta, artista y teórico conocido como el padre del llamado Nuevo Cine Latinoamericano, en el Manifiesto de Santa Fe plantea que la función del documental social en Latinoamérica es

testimoniar cómo esta realidad -esta subrealidad, esta infelicidad- la niega. Reniega de ella. La denuncia. La enjuicia, la critica, la desmonta. Porque muestra las cosas como son, irrefutablemente, y no como querríamos que fueran (o como nos quieren hacer creer-de buena o mala fe-que son). (p. 13)

Esta huella de identidad puede rastrearse asimismo en los formatos interactivos, en ocasiones mencionados como webdocs, idocs, documentales multimedia interactivos. Incluso en proyectos transmedia -de corte documental o periodístico- y 
en documentales inmersivos: aquellos que exploran las capacidades de las tecnologías $360^{\circ}$ y la realidad virtual.

A lo largo de la extensa geografía de América Latina es posible reconocer algunos polos de producción muy activos en la búsqueda de formatos innovadores y en la experimentación con nuevas tecnologías para narrar historias del orden de lo real. Las contribuciones recibidas para el presente número de Hipertext.net nos han permitido mapear el estado de la cuestión, historizando sus producciones, analizando sus características intrínsecas, revisando sus experiencias de diseño narrativo, circulación y consumo, e identificando incluso los desafíos y oportunidades que se presentan para la no-ficción interactiva y transmedia en esta región.

Entre los/as investigadores/as y productores/as que sumaron su mirada a esta edición se reconocen aportes de varios países. Argentina, Brasil, Colombia, España, México, Uruguay, se inscriben en este entramado de perspectivas que da cuenta del inmenso potencial creativo e innovador de una región que relata su propia historia desde diversas realidades, asumiendo la territorialidad como una dimensión posible (y necesaria) para narrar y experimentar. En muchas ocasiones, estos proyectos se articulan en torno a estrategias interactivas, participativas, colaborativas y co-creativas.

En el presente número, a partir del mapeo de la producción, análisis de contenido y estudio de cinco casos seleccionados, Jorge Vázquez Herrero (desde España), Lucila Benito (desde Argentina) y Natalia Revello Mouriz (desde Uruguay) consiguen poner en relieve la implicación de las instituciones universitarias en el desarrollo del documental interactivo y transmedia latinoamericano, así como la fuerte presencia de temáticas vinculadas al territorio, las comunidades y la denuncia social, propias del ámbito local.

Por su parte, la investigadora argentina María Celeste Marrocco se atreve a explorar profundamente los procesos de guionado de proyectos transmedia, buscando comprender cómo funciona el desarrollo de estrategias narrativas multiplataforma. Para ello, revisa una serie de casos locales, identifica un conjunto de regularidades en la metodología de diseño narrativo y propone conceptualizaciones que sistematizan estas prácticas creativas.

Verónica Torres recupera y desglosa la propuesta de narrativa transmedia de Experiencia Cortázar, sobre los detalles de los viajes que el escritor de "Rayuela" realizó por Argentina antes de su partida a Europa, a mediados de los 1940. En su artículo, la autora introduce el concepto de usuario "cómplice", considerando cómo los productores articularon las estrategias de lectura, las formas de abordaje y los recorridos a través de plataformas.

En este mismo sentido, Miguel Vilte presenta un análisis comparativo de los documentales transmedia Malvinas 30 y El feriante, identificando elementos y características de estas producciones y exponiendo las transformaciones que se produjeron en la construcción narrativa.

Entre las contribuciones brasileñas encontramos a Katia Maciel, quien nos ofrece un panorama de las producciones innovadoras desarrolladas en el marco del Programa de Posgraduación en Medios Creativos de la Universidad Federal de Rio de Janeiro. Experimentando con diversas tecnologías y técnicas narrativas, sus estudiantes han producido un conjunto de webdocs, videos interactivos, documentales $360^{\circ}$ y proyectos de realidad virtual de impacto y difusión nacional e internacional.

Carolina Gois Falandes y Denis Porto Renó, también investigadores brasileños, trascienden las fronteras de su país para acercarnos una cartografía de la no-ficción inmersiva iberoamericana, detectando 28 polos de producción que involucran a 7 países. En estos espacios resulta fundamental el aporte de las universidades públicas como engranaje que motoriza la innovación y la producción.

También desde Brasil, las investigadoras Debora Lopez y Aline Monteiro Homssi plantean que en el contexto de la llamada cultura del fan, las audiencias tradicionales de la radio se potencian a partir de la dinámica del consumo y expansión del lenguaje parasonoro del podcasting.

Nazly López Díaz, desde Colombia, nos invita a reflexionar en torno a la mediamorfosis del cine documental, considerándolo un género cuyas fronteras se encuentran en expansión. Para ello, propone primero una inmersión profunda por su historia, siempre asociada a las innovaciones tecnológicas que influyeron en su desarrollo. Luego analiza un conjunto de experiencias latinoamericanas donde las nuevas narrativas documentales asumieron formas gamificadas, interactivas e inmersivas.

El colombiano Carlos Obando Arroyave nos sugiere que el nuevo desafío narrativo latinoamericano, se da a partir de la construcción del relato de los documentales interactivos y transmedia y que la clave está en contar historias de forma local para poder ver de modo global. En su artículo analiza cuatro trabajos de diversas tipologías: inmersivos 360, transmedias e interactivos.

Por su parte, el mexicano Pablo Martínez Zárate, reconocido productor de obras interactivas y experimentales, recupera la experiencia desarrollada desde el Laboratorio Iberoamericano de Documental de la Universidad Iberoamericana Ciudad de México. A partir de un recorrido por los fundamentos que sostienen esa praxis, nos ofrece un conjunto de nociones y modelos de corte metodológico para el desarrollo de proyectos documentales transmedia. En este marco, el creador esgrime la urgencia del arte documental latinoamericano como expresión de un territorio de crisis cuya realidad herida 


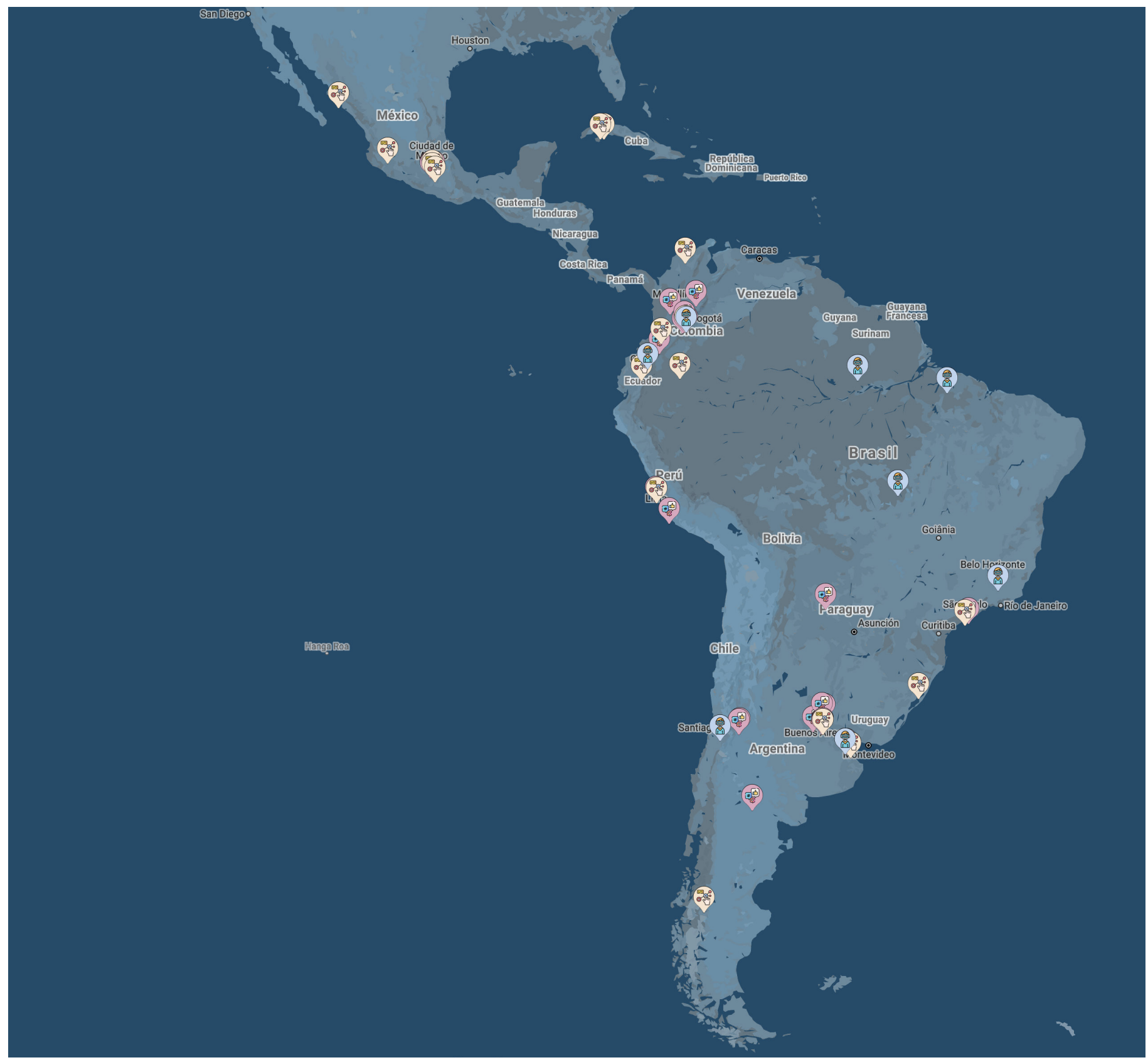

Figura 1. Cartografía de las nuevas narrativas de no-ficción latinoamericana.

nos obliga a reimaginar permanentemente nuestros horizontes de posibilidad.

Desde el estado de Jalisco en México, los investigadores Diego Zavala Scherer y Afra Citliali Mejía Lara, exponen el proyecto participativo desarrollado en San Juan de Ocotán, producido por un grupo de jóvenes surgidos a partir de un taller sobre alfabetización transmedia. Estos jóvenes se constituyeron en una productora, para narrar las historias de su barrio y su ciudad, para constituir una memoria colectiva cultural transmedial en contracara de la estigmatización social de esa comunidad por parte de los medios de comunicación.

En sintonía con los documentales sociales, desde Uruguay, Santiago López Delacruz, analiza dos casos de producciones de no-ficción, para ver el rol social, de visibilización y de empoderamiento de la comunidad LGBTQI, que promueve un proceso de autorrepresentación de las sexualidades diversas, desde la propia experiencia transmediática, como un espacio de identidad y de identificación desde la cultura participativa y la inteligencia colectiva.

La contribución española a este monográfico viene de la mano de José Luis Torres Martín, Andrea Castro Martínez y Pablo Díaz Morilla, que realizan un estudio de caso sobre el documental interactivo Metáfora Viva, 30 años de orden y caos de la Escuela Internacional de Cine y Televisión de San Antonio de los Baños (EICTV) de Cuba, donde analizan la transmedialidad, la interactividad y los rasgos que lo vincula al resto de producción documental interactiva realizada en Latinoamérica en la actualidad. 
El aporte de estos autores hizo posible una primera cartografía de proyectos documentales interactivos, inmersivos y transmedia gestados desde diversos espacios latinoamericanos. A este mapeo inicial hemos sumado otras producciones, igualmente reconocidas por sus valiosa contribución narrativa e innovadora, para completar la cartografía de las nuevas narrativas de no-ficción latinoamericana, un repositorio con más de 60 proyectos actualmente activos en América Latina.

Para el desarrollo cartográfico contamos con el enorme trabajo de Patricio Irisarri, miembro del equipo de la Dirección de Comunicación Multimedial de la Universidad Nacional de Rosario, docente del módulo Periodismo de datos y visualización de la información en la Maestría en Comunicación Digital Interactiva.

En el camino han quedado proyectos como Vibrato. Escuela Orquesta del Barrio Ludueña (2008), Peligro. Obras en construcción (2009) y Migraciones. Humedales del Paraná (2011), tres producciones de la serie DocuMedia. Periodismo Social Multimedia del \#DCMteam (Universidad Nacional de Rosario, Argentina). También Proyecto Walsh (2010) y \#Malvinas30 (2012), dirigidos por Álvaro Liuzzi, Río de Janeiro - Autorretrato (2011), de Marcelo Bauer, Sérgio Moraes, Giovanni Francischelli y Lucian Rosa y el proyecto colombiano El charco azul (2013), de Irene Lema. En buena medida, Las mutaciones evolutivas del ecosistema de medios y las transformaciones de las tecnologías disponibles para producir y consumir contenidos digitales los han dejado hoy inaccesibles. Es por ello que estos proyectos no fueron incorporados en la edición final del mapa, aunque no queríamos dejar de mencionarlos aquí como pioneros en las nuevas narrativas de no-ficción.

Como todo proyecto en construcción, esta cartografía está abierta a nuevas incorporaciones de obras. Por eso invitamos a los/as lectores/as a enviar enlaces de aquellos trabajos no referenciados en el mapa al correo de los editores.

Cabe destacar que muchas de las producciones mencionadas en los artículos y la cartografía resultaron finalistas o ganadoras de las primeras dos ediciones del Festival Internacional de Nuevas Narrativas de No Ficción (FINNOF), un espacio que busca galardonar proyectos en categorías que contemplan lenguajes y formatos narrativos emergentes, como el documental interactivo, la serie web, el documental transmedia, los proyectos de realidad virtual y los podcasts.

En estos formatos, la no-ficción latinoamericana tiene aún muchas historias por contar y un largo camino por seguir recorriendo, en busca de su estatuto, con ambición innovadora, consolidando una identidad digital propia, diversa, colectiva y políticamente comprometida con las problemáticas propias de su territorio y de su tiempo.

\section{Referencias}

Birri, F. (1964). La Escuela Documental de Santa Fe. Una experiencia piloto contra el subdesarrollo cinematográfico en Latinoamérica. Documentos del Instituto de Cinematografía de la Universidad del Litoral. Santa Fe: UNL.

Cartografía de las nuevas narrativas de no ficción latinoamericana: https://www.google.com/maps/d/embed?mid=1TZI5sds_zCadRIARpzW5x_6u6XyFhBx8cthl=es

Festival Internacional de Nuevas Narrativas de No Ficción (FINNOF): http://finnof.org/

\section{CV}

Fernando Irigaray. Magister en nuevas tecnologías de la información y de la comunicación (UNED-España), licenciado en comunicación social y candidato a doctor en comunicación social, en la Universidad Nacional de Rosario (UNR). Director de la Maestría en Comunicación Digital Interactiva y de Comunicación Multmedial en la UNR. También es director ejecutivo de la Cátedra Latinoamericana de Narrativas Transmedia (ICLA-UNR). Fue miembro del comité académico de la cátedra de tecnopolítca y cultura digital del Centro Internacional de Estudios Superiores de Comunicación para América Latina (CIESPAL). Dirige y produce programas y documentales para TV, interactivos y transmedia y cuenta con una extensa producción editorial. Ha recibido numerosos premios y reconocimientos nacionales e internacionales.

Anahí Lovato. Magister en Comunicación Digital Interactiva y Licenciada en Comunicación Social (UNR) y candidata a doctora por la Universitat Pompeu Fabra (España). Coordinadora de contenidos multimedia en la Dirección de Comunicación Multimedial (UNR). Guionista transmedia, multimedia y audiovisual en \#DCMteam. Miembro del Comité Ejecutivo de la Cátedra Latinoamericana de Narrativas Transmedia (ICLAUNR). Docente en UNR, UNC, EPCTV, ISET No18 y ETER. Publicó libros y artículos sobre narrativas transmedia y periodismo digital. 